\title{
COMPARISON OF MEASUREMENTS OF ANGULAR HADRON ENERGY SPECTRA, INDUCED ACTIVITY AND DOSE WITH FLUKA82 CALCULATIONS
}

Graham R.Stevenson, Pertti A.Aarnio*, Alberto Fassò, Johannes Ranft $\dagger$, Jorma V.Sandberg* and Peter Sievers.

\author{
CERN, Geneva, Switzerland
}

\begin{abstract}
Three experiments are described in which extended targets were bombarded with high-energy protons. The experiments were simulated using the Monte-Carlo hadron cascade package FLUKA82. The measured and simulated quantities included the hadron yield around a target as a function of polar angle and energy and the two-dimensional $(r-z)$ distributions of stars and energy deposition inside an aluminium cylinder. The agreement between the calculations and measurements is generally within experimental errors.
\end{abstract}

Submitted to Nuclear Instruments and Methods in Physics Research

* Helsinki University of Technology, Espoo, Finland

$\dagger$ Karl Marx University, Leipzig, G.D.R. 


\section{INTRODUCTION}

The purpose of the comparisons described here was to test the overall performance of the FLUKA82 hadronic cascade code [1] against experiments which involve the development of the hadronic cascade in extended targets, and thus include the physics of particle transport and of the development of the electromagnetic cascade as well as the physics of particle production. The performance of the particle production models is described elsewhere [2]. The present comparisons were designed to test features of the standard version of FLUKA82 without any user-written additions, i.e. the only estimators included were star densities, fluences and energy densities. All comparisons were made on an absolute basis and no parameters were specially adjusted in these calculations.

The comparisons involved differential properties of the cascade which are a more severe test than a comparison of integral quantities alone. The three-dimensional development of the cascade as well as the angular and energy spectra of the secondary hadrons were calculated and measured. These properties provide very critical tests of the physics in a cascade simulation because they need the reproduction of the correct forward:backward ratio of the produced secondaries as well as the correct treatment of the low-energy secondaries produced in intranuclear cascades.

The experiments themselves were designed to provide information on quantities of interest for radiation protection. It is necessary, for instance, to know the hadron flux above a given threshold as a function of angle around a target bombarded by a proton beam in order to predict the damage to and radioactivation of components near ejection septa. This type of experiment is described in section 2 of this paper and more completely in [3]. Because of the need to know the activation of the material as well as the heating effect and the radiation damage caused by high energy hadronic cascades, the experiments measuring the activation and dose deposition inside an aluminium block were performed [4]. These experiments are described in sections 3 and 4.

\section{HADRON FLUENCE SPECTRA}

A $15 \mathrm{~cm}$ long copper target having a diameter of $1.3 \mathrm{~cm}$ was irradiated with $225 \mathrm{GeV} / \mathrm{c}$ protons at the CERN SPS [3]. Spallation detectors were used to measure the hadron fluence [5]. These were cubic blocks of copper with a $2.5 \mathrm{~cm}$ edge in which the activities of certain gamma-emitting radio-isotopes produced during the irradiation were assayed. Twelve detectors were located at a distance of $38 \mathrm{~cm}$ from the centre of the target at angles from $10^{\circ}$, to $150^{\circ}$ with respect to the incident beam. The spallation reactions used are shown in Table 1.

The energy dependent spallation reaction cross-sections were calculated using the semiempirical cross-section formulae of Rudstam [6] or Silberberg and Tsao [7]. The Silberberg-Tsao formulae were used only for the $\mathrm{Cu}-{ }^{7} \mathrm{Be}$ cross-section calculations where the Rudstam formulae are not applicable. The effective cross-section was calculated from the energy dependent cross-sections using a constant weighting spectrum which is justified when the cross-sections have a clear threshold behaviour. The threshold energy was taken as the point where the cross-section first reaches $1 \%$ of the maximum value. The effective cross-sections and the threshold energies used are shown in Table 1. By using the effective cross-sections, the measured spallation product activities were converted to hadron fluences at each angle. The results were normalized to one incident proton.

The fluences measured by the $\mathrm{Cu}-{ }^{56} \mathrm{Co}$ reaction were compared with those from the ${ }^{27} \mathrm{Al}-{ }^{18} \mathrm{~F}$ and ${ }^{12} \mathrm{C}-{ }^{7} \mathrm{Be}$ reactions in aluminium and polyethylene disks respectively, which also have an effective threshold of $35 \mathrm{MeV}$ and effective cross-section of $8 \mathrm{mb}$ and $11 \mathrm{mb}$ [3]. These cross-sections are derived from measurements rather than calculations. There is complete agreement on an absolute basis between the yields as measured by the three techniques, as can be seen in Figure 1. 


\section{Table 1:}

Effective threshold energies and spallation yield cross-sections from copper as calculated with the Rudstam CDMD formulae.

\begin{tabular}{llc} 
Product & $\begin{array}{c}\text { Threshold } \\
\text { energy } \\
(\mathrm{MeV})\end{array}$ & Cross-section \\
& & $(\mathrm{mb})$ \\
${ }^{56} \mathrm{Co}$ & 35.7 & 5.2 \\
${ }^{54} \mathrm{Mn}$ & 50.0 & 27.1 \\
${ }^{52} \mathrm{Mn}$ & 66.6 & 7.2 \\
${ }^{51} \mathrm{Cr}$ & 74.8 & 21.1 \\
${ }^{48} \mathrm{~V}$ & 105.0 & 9.1 \\
${ }^{46} \mathrm{Sc}$ & 126.0 & 8.1 \\
${ }^{24} \mathrm{Na}$ & 393.0 & 2.4 \\
${ }^{7} \mathrm{Be}$ & 561.0 & $6.7^{*}$ \\
\multicolumn{2}{c}{} &
\end{tabular}

The experiment was simulated using FLUKA82. All hadrons above given energy thresholds leaving the target were scored as a function of polar angle. The energy thresholds used were $50 \mathrm{MeV}$, $70 \mathrm{MeV}, 126 \mathrm{MeV}, 393 \mathrm{MeV}$ and $561 \mathrm{MeV}$, corresponding to the thresholds in Table 1. The experimental and the simulated results are compared in Figure 2. Note that only the statistical errors in the calculation are shown in this Figure. The calculated values were derived from only one cascade simulation, i.e. the same histories have been used for each threshold energy. Thus any bumps seen in curves at the same angle for different thresholds have no systematic meaning but are the result of using the same histories. The measurements and calculations are all absolute without any normalization.

As can be seen in Figure 2 the calculations and the experimental measurements in general agree even in the backward direction. The experimental points are, however, systematically on the low side when compared with the calculations. This can be understood if it is realized that the true threshold energies could be somewhat higher than those assumed (the $1 \%$ point was used here, as in the reference [5] in order to facilitate comparison with earlier experiments [8]). This would have the effect of lowering the calculated curves which should be used for comparison in Figure 2 without affecting the measured values.

The cross-sections also carry uncertainties of the order of $30 \%$ for incident protons as well as a dependence on initial hadron type, which is not known. This, taken together with the measurement errors of a few percent, means that the calculations can be considered to agree with the measurements to within the expected errors.

It should be emphasized that practically all particles leaving the target at angles above $90^{\circ}$ come from the intranuclear cascade. This is correctly modelled in FLUKA82. Care should be taken in using any model which includes only hadron-hadron collisions and simulates hadron-nucleus collisions 
only by a simple multiplicity enhancement, neglecting the intranuclear cascading. Such models cannot be expected to reproduce correctly experimental results such as those of Figure 2, as was in fact found by running FLUKA82 without the correct intranuclear cascade model.

\section{STAR DENSITIES}

One of the basic quantities which can be scored in FLUKA82 is the density of inelastic interactions, i.e. the number of stars per $\mathrm{cm}^{3}$ per incident particle: this quantity is closely related to the density of radioactive nuclei produced. The experimental set-up indicated in Figure 3 was used to provide a comparison with Monte-Carlo predictions of the 3-dimensional star-density distribution.

An aluminium cylinder $134 \mathrm{~cm}$ long by $11.5 \mathrm{~cm}$ radius was irradiated with a pencil beam of 200 $\mathrm{GeV} / \mathrm{c}$ protons. After the irradiation, circular samples were punched out of the aluminium detector plates at different axial and radial positions. The saturation activities of ${ }^{18} \mathrm{~F}$ was determined in these samples by standard gamma spectrometric techniques using a $3^{\prime \prime} \times 3^{\prime \prime}$ sodium iodide crystal. As mentioned above, this reaction can be taken to have an effective threshold at $35 \mathrm{MeV}$. The beam intensity was monitored placing a pack of three thin aluminium foils in front of the cylinder. The saturation activity of the ${ }^{18} \mathrm{~F}$ in the centre foil was also measured.

In order to make the comparison less sensitive to the inaccuracies in the cross-sections, the measured activities and calculated star densities were not compared directly but each was referred to its value at the origin of the cascade in the following manner.

The saturation activity per unit volume of ${ }^{18} \mathrm{~F}$ in an aluminium sample at a position $(\mathrm{r}, \mathrm{z})$ in the cylinder is given by:

$$
\mathrm{a}(\mathrm{r}, \mathrm{z})=(\rho \mathrm{L} / \mathrm{A}) \Sigma_{\mathrm{i}} \int \sigma_{\mathrm{i}}(\mathrm{E}) \phi_{\mathrm{i}}(\mathrm{E}, \mathrm{I}, \mathrm{z}) \mathrm{dE} / \mathrm{t},
$$

where $\mathrm{L}$ is Avogadros number, $\rho$ and $\mathrm{A}$ are the density and atomic weight of aluminium respectively, the sum $\Sigma$ is taken over all particle types $i$, the ${ }^{18} \mathrm{~F}$ production cross-section is $\sigma_{i}$ and the differential fluence spectrum of particles in the cascade is $\phi_{i}$. The effective irradiation time is $t$.

Equation (1) can be simplified:

$$
\mathrm{a}(\mathrm{r}, \mathrm{z})=(\rho \mathrm{L} / \mathrm{A}) \sigma_{\mathrm{f}} \Phi_{\mathrm{f}}(\mathrm{r}, \mathrm{z}) / \mathrm{t},
$$

where $\sigma_{\mathrm{f}}$ is the cascade-averaged ${ }^{18} \mathrm{~F}$ production cross-section (assumed to be independent of position) and $\Phi_{\mathrm{f}}$ is the hadron fluence above the ${ }^{18} \mathrm{~F}$ production threshold.

The saturation activity per unit thickness in the beam foil is given by:

$$
a_{0}=(\rho L / A) \sigma_{f}\left(E_{0}\right) N / t
$$

where $\sigma_{\mathrm{f}}\left(\mathrm{E}_{0}\right)$ is the ${ }^{18} \mathrm{~F}$ production cross-section by protons of energy $\mathrm{E}_{0}$ and $\mathrm{N}$ is the total number of protons passing through the foil.

Thus:

$$
a(r, z) / a_{0}=\left[\sigma_{f} \Phi_{f}(r, z)\right] /\left[\sigma_{f}\left(E_{0}\right) N\right]
$$


Similar expressions can be obtained using the star densities calculated by FLUKA82 in the aluminium cylinder:

$$
s(r, z)=(\rho L / A) \sigma_{s} \Phi_{s}(r, z),
$$

where $\sigma_{\mathrm{s}}$ is the average star-production cross-section (again assumed to be independent of position) and $\Phi_{s}(r, z)$ is the star-producing hadron fluence. The number of stars produced per unit thickness in the beam foil is:

$$
\mathrm{s}_{0}=(\rho \mathrm{L} / \mathrm{A}) \sigma_{\mathrm{s}}\left(\mathrm{E}_{0}\right) \mathrm{N},
$$

where $\sigma_{s}\left(E_{0}\right)$ is the star-production cross-section at the proton beam energy.

As before

$$
s(r, z) / s_{0}=\left[\sigma_{s} \Phi_{s}(r, z)\right] /\left[\sigma_{s}\left(E_{0}\right) N\right]
$$

Figure 4 compares plots of the quantities $a(r, z) / a_{0}$ and $s(r, z) / s_{0}$ as a function of radius for different depths in the aluminium cylinder. If it can be assumed that the cascade-averaged ${ }^{18} \mathrm{~F}$ production cross-section, normalized to the cross-section at the proton beam energy, is identical to that of the cascade-averaged star-production cross-section, also normalized to the star-production cross-section at the beam energy, i.e. that

$$
\sigma_{f} / \sigma_{f}\left(E_{0}\right)=\sigma_{s} / \sigma_{s}\left(E_{0}\right),
$$

then, from equations (4) and (7), Figure 4 must be a direct comparison of $\Phi_{f}$ and $\Phi_{s}$. It will be seen from this figure that the measured fluence is somewhat higher than that predicted from the star densities. This is to be expected since the ${ }^{18} \mathrm{~F}$ production threshold is approximately $35 \mathrm{MeV}$, whereas the lowest energy considered in the cascade simulation is $50 \mathrm{MeV}$. Furthermore the difference in Figure 4 is approximately the same as the difference in the fluence at large angles for 35 and $50 \mathrm{MeV}$ taken from Figures 1 and 2.

On the assumption that the ratios in equation (8) are equal to unity, then the ratios plotted actually correspond to the average hadron fluence per incident primary proton.

\section{DOSE}

The energy deposition density, dose, is also obtained as a standard option from FLUKA82. Data obtained during the same experiment as that described in the previous section [4] were used to compare with the longitudinal and transverse energy deposition densities calculated by the code. The dosimeter data were obtained from radio-photoluminescent (RPL) dosimeters mounted on the aluminium detector plates. These glass dosimeters have approximately the same effective atomic number, atomic weight and density as aluminium.

The results of the experiment together with the FLUKA82 simulations are shown in Figure 5. It should again be emphasized that the numbers quoted are absolute. Two ways of treating energy deposition from the electromagnetic cascade are available in the FLUKA82 program. The first involves a simple parameterization of the electromagnetic cascade [1]; the second calls the EGS code [9] whenever a photon or an electron is created in FLUKA82. The calculated data in Figure 5 refer to data obtained using the simple parameterization. In this particular simulation of the aluminium cylinder experiment, no significant differences were found when using the EGS code. 
When comparing the calculations and the experiments it is seen that the agreement is again better than within a factor of two. The experimental points themselves show an error of the same order (see, for instance, the separate measurements at a radius of $7 \mathrm{~cm}$ in Figure 5) The calculated values, however, seem to systematically underestimate the data.

Part of the discrepancy could be due to the approximations made in FLUKA82 for the production and transport of low-energy evaporation fragments, mainly neutrons. The energy of these particles is deposited around the interaction point in an approximate way (for details see [1]). These neutrons are responsible for a significant part of the energy deposited far away from the core of the cascade. Better approximations involving the inclusion of neutron removal cross-sections instead of inelastic cross-sections and the use of a more detailed evaporation model are currently being tested for future implementation in the FLUKA82 code.

\section{CONCLUSIONS}

In the comparisons described here, three quantities directly scorable by FLUKA82 were used: hadron yield, star densities and energy deposition densities. In the comparisons these quantities were summed for all particles even though FLUKA82 could have provided the star densities and hadron yield of any single particle type. The agreement with the experiments was generally within the experimental errors but some systematic differences could be seen. The experiments described here are, however, not sufficiently selective to pinpoint with certainty the approximations made in the FLUKA82 code which could be responsible for the remaining discrepancies. However, the good general agreement between the experimental data and the FLUKA82 simulations, in situations which involved both poorly and fully developed cascades, indicates that the FLUKA82 program can be used to predict quantities of interest for radiation protection with an accuracy which is more than sufficient for accelerator design purposes.

\section{ACKNOWLEDGEMENTS}

The authors wish to thank K.Goebel (RP Group, CERN) and B.de Raad (SPS Division, CERN) for their encouragement in this work. The help of B.de Raad in obtaining the necessary beam time during Machine Development Periods at the CERN SPS is gratefully acknowledged. We also wish to thank the Machine Operators of the SPS-AOP Group for their assistance. A final word of thanks must go to the technicians in the SPS Section of RP Group, especially A.Regelbrugge and F.Coninckx, for their participation in these experiments.

\section{REFERENCES}

[1] P.A.Aamio, J.Ranft and G.R.Stevenson, A long writeup of the FLUKA82 program, CERN Divisional Report TIS - RP/106-Rev. (1984).

[2] J.Ranf, P.A.Aamio, G.R.Stevenson, FLUKA82, CERN Divisional Report TIS - RP/156/CF (1985).

[3] G.R.Stevenson, A.Fassò, J.Sandberg, A.Regelbrugge, A.Bonifas, A.Muller, M.Nielsen, Measurements of the dose and hadron yield from copper targets in $200 \mathrm{GeV} / \mathrm{c}$ and $400 \mathrm{GeV} / \mathrm{c}$ extracted proton beams. An Atlas of the Results obtained, CERN Divisional Report TIS - RP/112 (1983).

[4] P.Sievers, Measurements of the energy deposition of 200 and $400 \mathrm{GeV} / \mathrm{c}$ protons in aluminium and copper, CERN Divisional Report SPS/ABT/77-1 (1977).

[5] J.Sandberg, Angular and energy distribution measurements of secondary hadron fluxes with multireaction spallation detectors at CERN SPS, Nucl. Instrum. and Meth. 200 (1982) 211.

[6] G.Rudstam, Systematics of spallation yields, Z. Naturforsch. 21a (1966) 1027. 
[7] R.Silberberg and C.H.Tsao, Partial cross-sections in high-energy nuclear reactions and astrophysical applications. I Targets with $Z \leq 28$; II Targets heavier than nickel, Astrophys. J. Suppl. 220, 25 (1973) 315.

[8] J.T.Routti, Angular distribution measurements of secondary-particle fluxes with spallation detectors and comparisons to hadron cascade calculations, Nucl. Sci. Eng. 55 (1974) 41.

[9] R.L.Ford, W.R.Nelson, The EGS code system: Computer programs for the Monte Carlo simulation of electromagnetic cascade showers, Stanford Linear Accelerator Center Report SLAC-210 (1978). 


\section{FIGURE CAPTIONS:}

Figure 1: $\quad$ Comparison of the measured hadron fluences as a function of polar angle using the spallation reaction $\mathrm{Cu}-{ }^{56} \mathrm{Co}(0)$, with ${ }^{27} \mathrm{Al}-{ }^{18} \mathrm{~F}\left({ }^{*}\right)$ and ${ }^{12} \mathrm{C}-{ }^{7} \mathrm{Be}$ reactions $(\nabla)$ all having an effective threshold energy of $35 \mathrm{MeV}$.

Figure 2: $\quad$ Comparison of experimental $(\nabla)$ and calculated $(+)$ hadron fluences above different energy thresholds as a function of polar angle around a copper target bombarded by $225 \mathrm{GeV}$ protons. For the details of the reactions at the different threshold energies, see Table 1 . The data have been multiplied by the factors shown before plotting.

Figure 3: Experimental set-up for the measurement of activation and energy deposition in an aluminium cylinder.

Figure 4: $\quad$ Comparison of experimental $(+)$ and calculated (histogram) hadron fluences using star densities inside an aluminium cylinder bombarded by $200 \mathrm{GeV}$ protons as a function of radius at different depths. The data have been multiplied by the factors shown before plotting.

Figure 5: $\quad$ Comparison of experimental $(+)$ and calculated (histogram) energy deposition densities inside an aluminium cylinder bombarded by $200 \mathrm{GeV}$ protons as a function of radius at different depths. The data have been multiplied by the factors shown before plotting. 


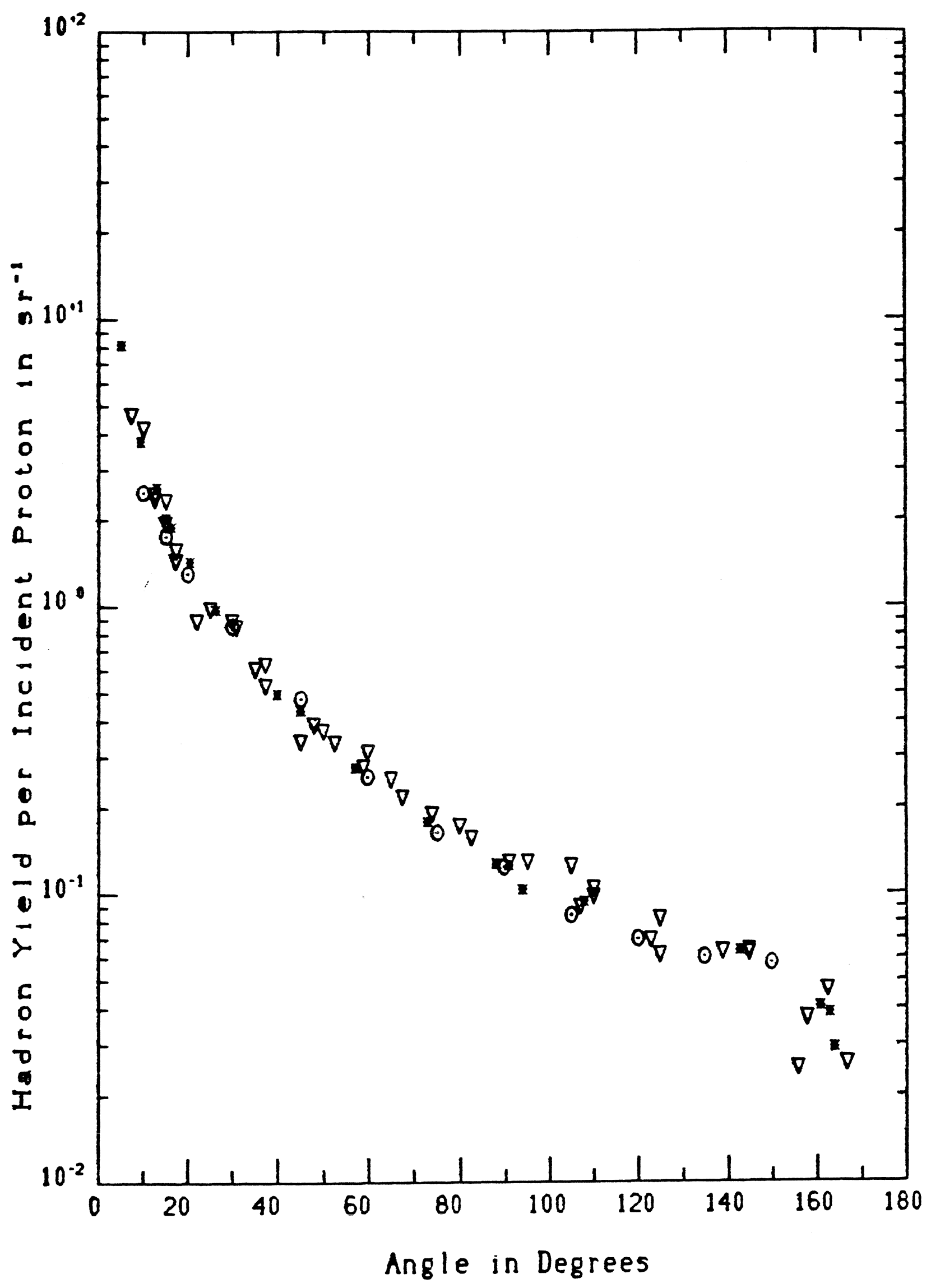

Fig. 1 


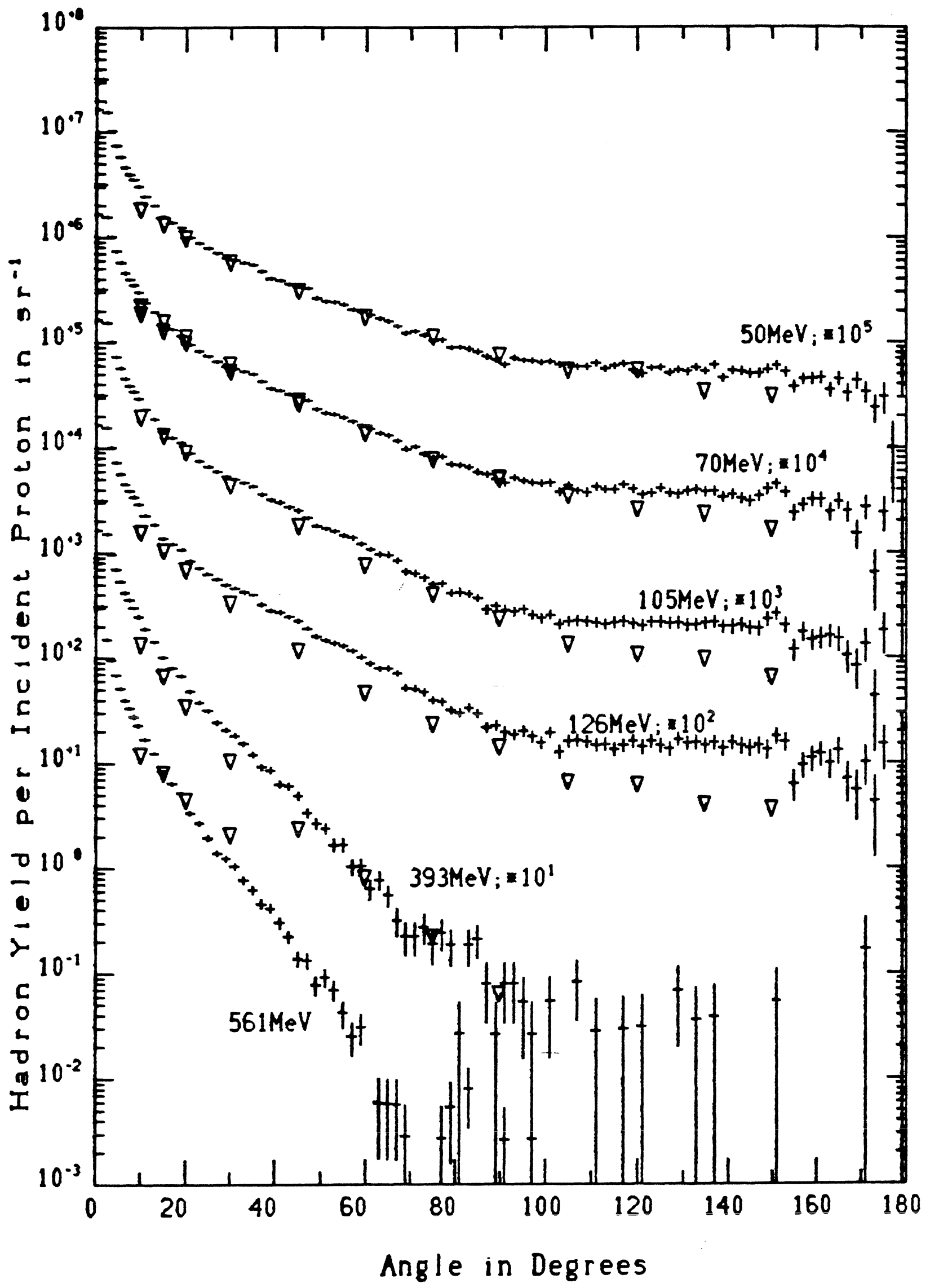

Fig. 2 


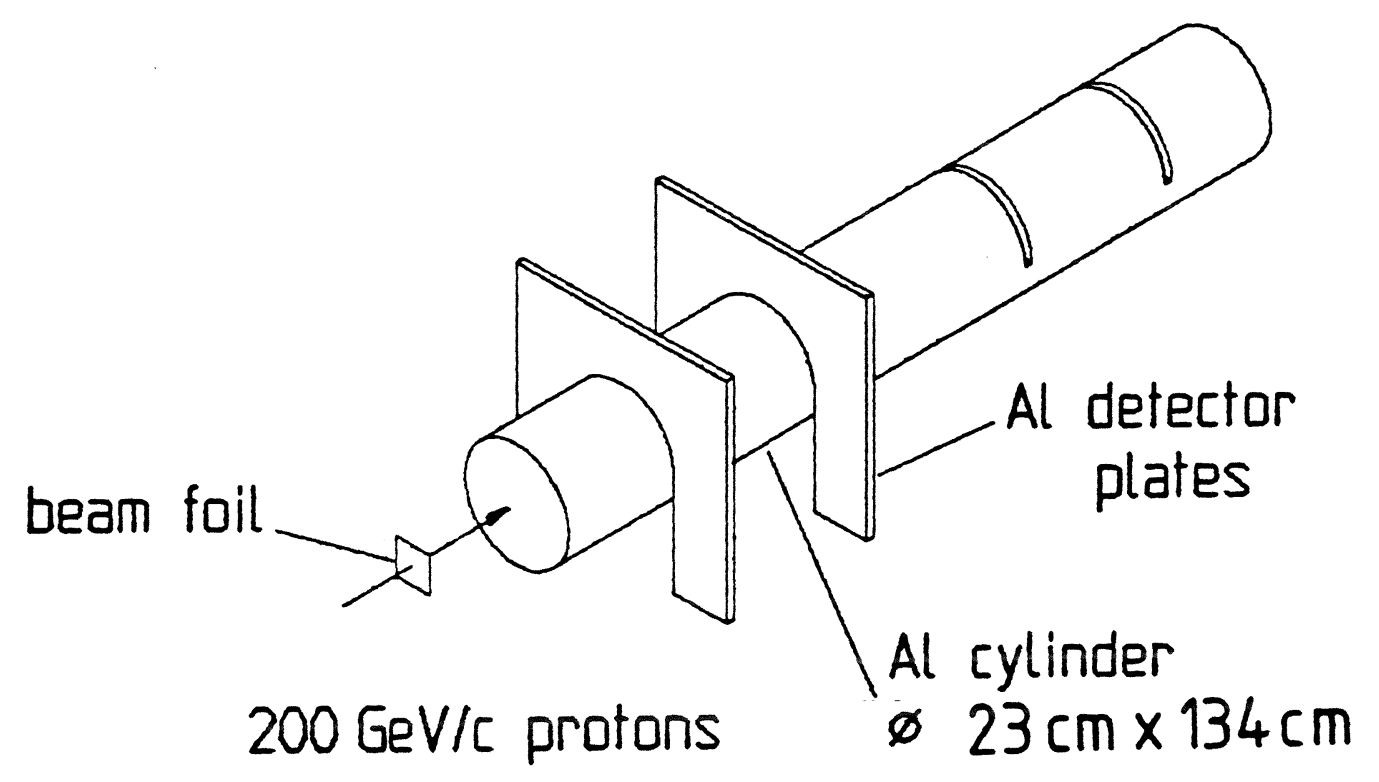

FIGURE 3 


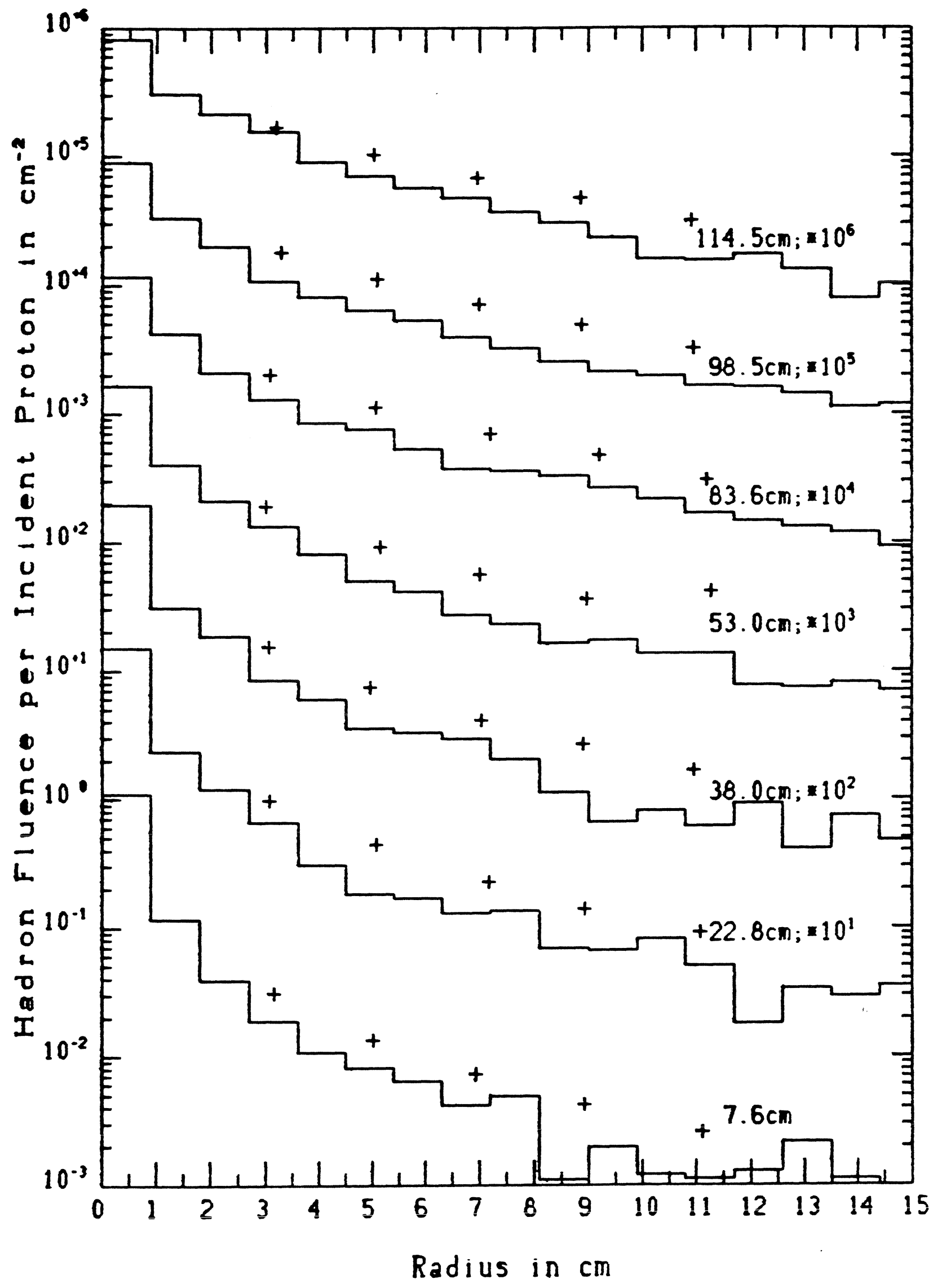

Fig. 4 


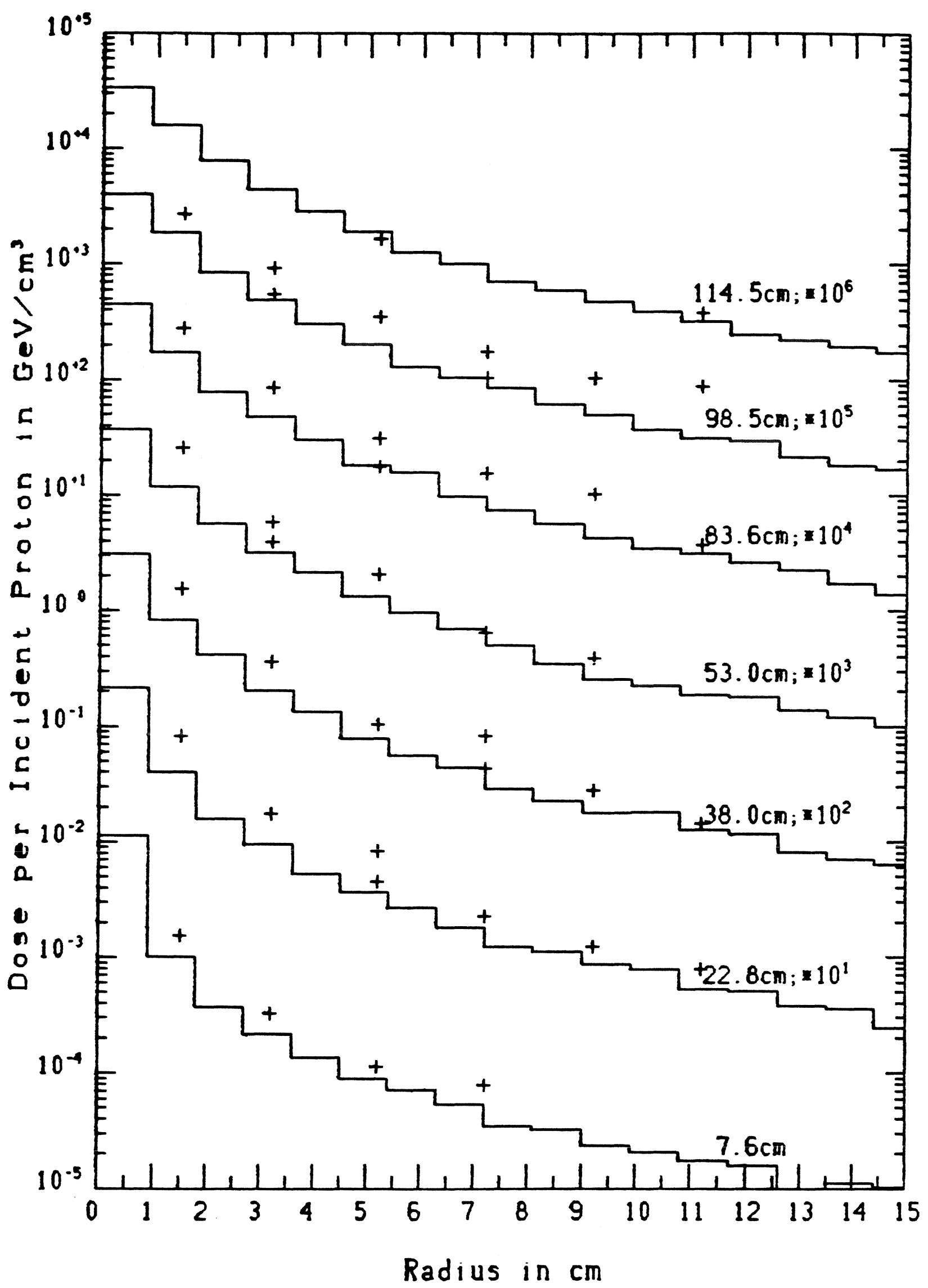

Fig. 5 